Neutron Resonance Radiography for Explosives Detection: Technical Challenges

W.L.Raas, B. Blackburn, E. Boyd, J.M. Hall, G. Kohse, R. Lanza, B. Rusnak, J.I.W. Watterson

November 11, 2005

IEEE Nuclear Science Symposium and Medical Imaging Conference

Fajardo, PR, United States

October 23, 2005 through October 29, 2005 
This document was prepared as an account of work sponsored by an agency of the United States Government. Neither the United States Government nor the University of California nor any of their employees, makes any warranty, express or implied, or assumes any legal liability or responsibility for the accuracy, completeness, or usefulness of any information, apparatus, product, or process disclosed, or represents that its use would not infringe privately owned rights. Reference herein to any specific commercial product, process, or service by trade name, trademark, manufacturer, or otherwise, does not necessarily constitute or imply its endorsement, recommendation, or favoring by the United States Government or the University of California. The views and opinions of authors expressed herein do not necessarily state or reflect those of the United States Government or the University of California, and shall not be used for advertising or product endorsement purposes.

This work was performed under the auspices of the U. S. Department of Energy by the University of California, Lawrence Livermore National Laboratory, under Contract No. W-7405-Eng.-48. 


\title{
Neutron Resonance Radiography for Explosives Detection: Technical Challenges
}

\author{
Whitney L. Raas, Member, IEEE, Brandon W. Blackburn, Member, IEEE, Erin Boyd, James Hall, Gordon Kohse, \\ Member, IEEE, Richard C. Lanza, Member, IEEE, Brian Rusnak, and John I. W. Watterson
}

\begin{abstract}
Fast Neutron Resonance Radiography (NRR) has recently become a focus of investigation as a supplement to conventional $x$-ray systems as a non-invasive, non-destructive means of detecting explosive material concealed in checked luggage or cargo containers at airports. Using fast (1-6 MeV) neutrons produced by the $D(d, n)^{3} H e$ reaction, NRR provides both an imaging capability and the ability to determine the chemical composition of materials in baggage or cargo. Elemental discrimination is achieved by exploiting the resonance features of the neutron cross-section for oxygen, nitrogen, carbon, and hydrogen. Simulations have shown the effectiveness of multiple-element NRR through Monte Carlo transport methods; this work is focused on the development of a prototype system that will incorporate an accelerator-based neutron source and a neutron detection and imaging system to demonstrate the realistic capabilities of NRR in distinguishing the elemental components of concealed objects. Preliminary experiments have exposed significant technical difficulties unapparent in simulations, including the presence of image contamination from gamma ray production, the detection of low-fluence fast neutrons in a gamma field, and the mechanical difficulties inherent in the use of thin foil windows for gas cell confinement. To mitigate these concerns, a new gas target has been developed to simultaneously reduce gamma ray production and increase structural integrity in high flux gas targets. Development of a neutron imaging system and neutron counting based on characteristic neutron pulse shapes have been investigated as a means of improving signal to noise ratios, reducing irradiation times, and increasing the accuracy of elemental determination.
\end{abstract}

\section{INTRODUCTION}

$\mathrm{D}$ ETECTION of conventional explosives remains a challenge to air security, as indicated by new requirements that mandate screening $100 \%$ of checked luggage at airports and the recent reports detailing lapses in security screening. Current explosive detection imaging systems rely on x-rays to distinguish dangerous material from innocuous items. The advantage of using neutrons as the probing radiation is the element-specific transmission probability, rather than densityspecific as is the case with $\mathrm{x}$-rays. The dependence of neutron attenuation on the cross-section of individual elements allows

Manuscript received October 11, 2005. This work was supported by the US Government, Technical Support Working Group and by the Transportation Security Administration.

W. Raas, B. Blackburn, E. Boyd, G. Kohse, and R. Lanza, are with the Department of Nuclear Science and Engineering at the Massachusetts Institute of Technology, Cambridge, MA 02139 USA (telephone: 617-253-5730, email: wraas@mit.edu).

J. Hall and B. Rusnak are with Lawrence Livermore National Laboratory, 7000 East Ave., MS L050 Livermore, CA, 94550.

$\mathrm{J}$. Watterson is with the Department of Nuclear Science and Engineering at the Massachusetts Institute of Technology, Cambridge, MA 02139 USA, on leave from the University of Witwatersrand, Johannesburg, South Africa. one to use NRR to obtain an elemental "map" of an object that details the amount of carbon, oxygen, nitrogen, and hydrogen present. Since most explosives have a characteristic amount of nitrogen and oxygen quite different from that of common items, explosive material can be separated from other objects based on elemental composition, leading to fewer false alarms [1]. The fundamental principle of NRR is the exploitation of resonance features of the total attenuation neutron crosssection in the 1-6 MeV range [2, 3]. Each element has a unique total cross-section that determines the probability that an incoming neutron of a particular energy will be scattered, absorbed or transmitted, as shown in Fig. 1.

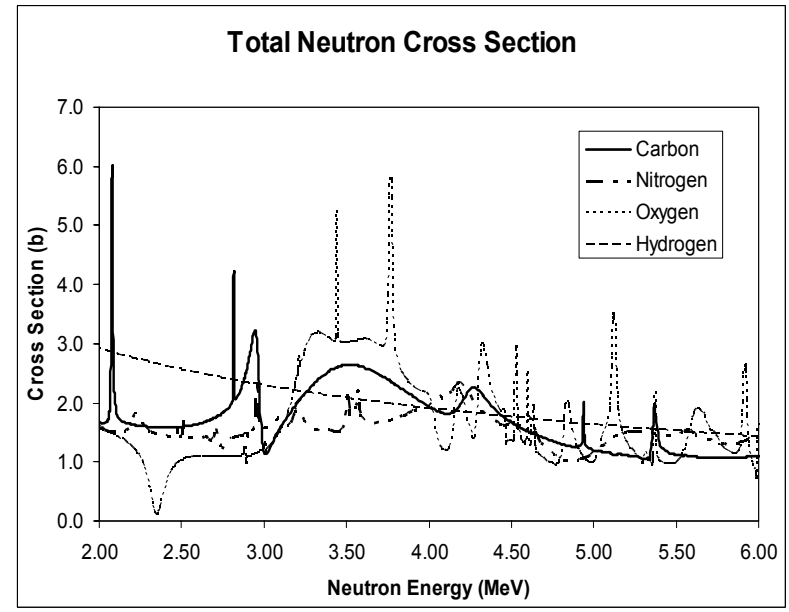

Fig. 1. Total neutron cross-section for $\mathrm{C}, \mathrm{N}, \mathrm{O}$, and $\mathrm{H}$.

An area where the cross-section for a particular element is high signifies that more incoming neutrons will be scattered than in areas where the cross-section for that element is low. For example, the value of the cross-section for oxygen in the peak at $3.25-3.75 \mathrm{MeV}$ is about thirty times that in the sharp valley at $2.4 \mathrm{MeV}$; consequently, the interaction probability is approximately thirty times higher at $2.4 \mathrm{MeV}$ than at $3.5 \mathrm{MeV}$. A two-dimensional image of an object can be formed at each of these energies using a scintillation detector coupled to a CCD camera. The difference between the two images results in a quantitative assessment of the amount of oxygen in the object under investigation.

The relative amount of multiple elements can be determined simultaneously by determining a series of neutron energies corresponding to at least one peak and one valley in the attenuation cross-section for each element of interest. For example, suppose we are interested in the elemental composition of an object containing a number $n$ different elements of interest. A neutron radiograph of the object is 
taken at a number $m$ different neutron energies, where $m \geq n$ and we construct a series of equations for each pixel:

$$
\begin{gathered}
a_{1,1} x_{1}+a_{1,2} x_{2}+a_{1,3} x_{3}+\ldots+a_{1, n} x_{n}=b_{1} \\
a_{2,1} x_{1}+a_{2,2} x_{2}+a_{2,3} x_{3}+\ldots+a_{2, n} x_{n}=b_{2} \\
a_{3,1} x_{1}+a_{3,2} x_{2}+a_{3,3} x_{3}+\ldots+a_{3, n} x_{n}=b_{3} \\
\vdots \\
a_{i, 1} x_{1}+a_{i, 2} x_{2}+a_{i, 3} x_{3}+\ldots+a_{i, j} x_{j}=b_{j} \\
\vdots \\
a_{m, 1} x_{1}+a_{m, 2} x_{2}+a_{m, 3} x_{3}+\ldots+a_{m, n} x_{n}=b_{m}
\end{gathered}
$$

where $x_{j}$ is the thickness of the element in question (in say $\left.\mathrm{g} / \mathrm{cm}^{2}\right), \mathrm{a}_{\mathrm{i}, \mathrm{j}}$ is the mass attenuation coefficient at energy $i$ for element $j$, and $b_{\mathrm{j}}$ is the measured intensity of the pixel. Equation (1) can be solved through a least-squares methods for the elemental content $x_{j}$, resulting in a pixel-by-pixel twodimensional map of elemental content.

\section{COMPONENTS OF AN NRR SYSTEM}

The system we propose would employ an accelerator-based $\mathrm{D}(\mathrm{d}, \mathrm{n})^{3} \mathrm{He}$ reaction as a neutron source:

$$
D+d \rightarrow n+{ }^{3} \mathrm{He}+3.27 \mathrm{MeV} .
$$

This reaction has a number of advantages for NRR, among them the lack of gamma rays produced directly in the reaction, the exothermic nature of the reaction, and the favorable kinematics of the reaction which produces neutrons with an angle-dependent energy relationship (Fig. 2). In order to take advantage of the full range of energies between 1 and $6 \mathrm{MeV}$ where resonance features in the cross-sections for light elements are most prevalent, deuterium ions are accelerated to an energy of $2.5 \mathrm{MeV}$ into a 4 atm deuterium gas target. The neutrons produced span an energy range from $5.13 \mathrm{MeV}$ at $0^{\circ}$ to $1.67 \mathrm{MeV}$ at $180^{\circ}$. By rotating the object under investigation around the neutron source, different neutron energies are made available. If the object, or the portion of the object being imaged, spans an angle less than ten degrees, the neutron beam can be considered effectively mono-energetic within the cone subtended by the object.

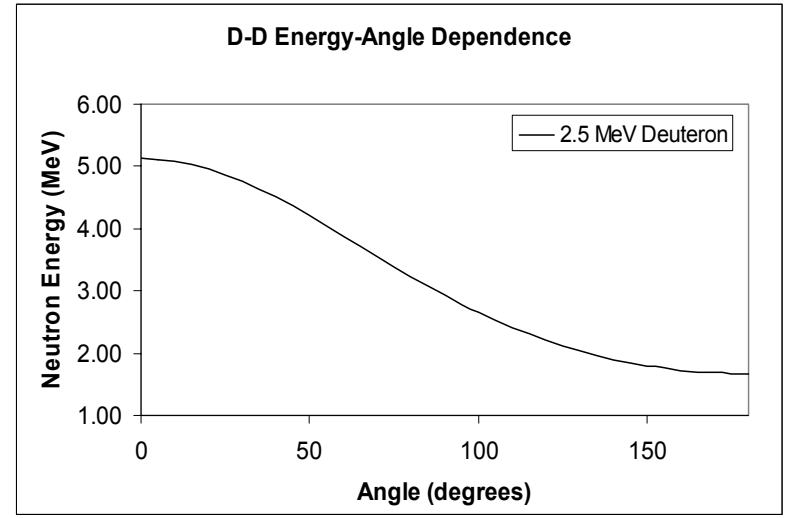

Fig. 2. $\mathrm{E}_{\mathrm{n}}$ angular dependence for the D-D reaction.

The neutron detection system, consisting of a light-emitting neutron detector such as solid plastic scintillator, coupled to a CCD camera for imaging purposes or to a light guide and photomultiplier tube (PMT) for neutron/gamma discrimination, is placed directly in line with the object under investigation and is not allowed to shift relative to the object. The neutron detection system is fully enclosed in a light-tight box to reduce extraneous light. To make a measurement of the neutron attenuation through an object at different energies, the object-detector assembly is rotated around the neutron source.

\section{SiMULATIONS AND EXPERIMENTS}

Specialized experiments involving a single element of interest and simulations of multiple-element objects have shown NRR to be viable as a non-invasive and non-destructive means of elemental determination of bulk material $[4,5]$. However, experimental multiple-element NRR has produced mixed results. Preliminary experiments using a D-D reaction for neutron production and probing items of known composition were performed to test the ability of NRR to discriminate between multiple elements in a sample of objects. The tandem accelerator at Ohio University was used to provide a $5 \mu \mathrm{A}, 2.5 \mathrm{MeV}$ deuteron beam for neutron production from a small deuterium gas target. The gas target held $3 \mathrm{~atm}$ of deuterium, separated from the accelerator beam line by a $5 \mu \mathrm{m}$ thin tungsten foil and utilizing a solid gold beamstop to stop the deuteron beam.

Attenuation coefficients $\left[\mathrm{a}_{\mathrm{i}, \mathrm{j}}\right.$ in (1)] were determined using four calibration objects: graphite for carbon, polyethylene for hydrogen, water for oxygen, and melamine for nitrogen. One blank, unobstructed radiograph was taken to determine the intensity of each pixel due to the unattenuated neutron flux. Radiographs were taken at ten neutron energies $(2.36,2.88$, $3.08,3.21,3.55,3.76,4.11,4.31,4.87$, and $5.13 \mathrm{MeV}$ ); the linear attenuation coefficients, $\mu$, for each element at each energy were calculated using the detected intensity of each pixel. The mass attenuation coefficients, $a_{i, j}$ are determined simply by $\mathrm{a}_{\mathrm{i}, \mathrm{j}}=\mu_{\mathrm{i}, \mathrm{j}} / \rho_{\mathrm{i}}$, where $\rho_{\mathrm{i}}$ is the density of the calibration material.

Eight test objects were radiographed with neutrons of the same energy as the calibration objects: water, ammonium nitrate, acetone, toluene, melamine, methanol, sugar, and polyethylene. The material was contained in small HDPE 
plastic bottles and included only items of known composition containing only the elements $\mathrm{H}, \mathrm{C}, \mathrm{O}$, and $\mathrm{N}$ in order to determine the accuracy of the results. The objects were placed in the neutron beam for 30 minutes and the images recorded by a liquid-nitrogen-cooled CCD camera with a 1024 x 1024 array of $24 \mu \mathrm{m}$ pixels. The quality of the images was further improved by post processing to remove "dead" pixels and the effects of cosmic rays. The elemental areal density $\left(\mathrm{g} / \mathrm{cm}^{2}\right)$ was determined by using a Householder transformation to solve for $\mathrm{x}_{\mathrm{i}}$ in (1), resulting in a value for the areal density of $\mathrm{H}, \mathrm{C}, \mathrm{O}$, and $\mathrm{N}$ in each pixel.

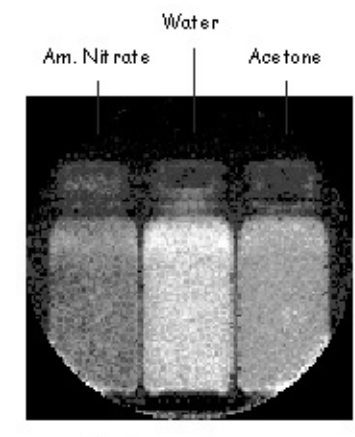

Hydrogen happ ing (areal density)

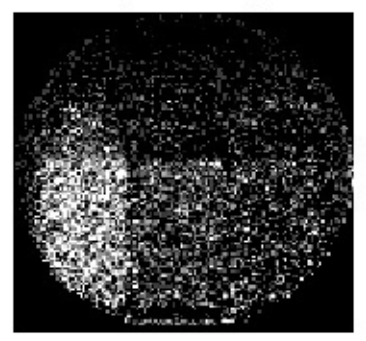

Nitrogen Mapping (areal density)

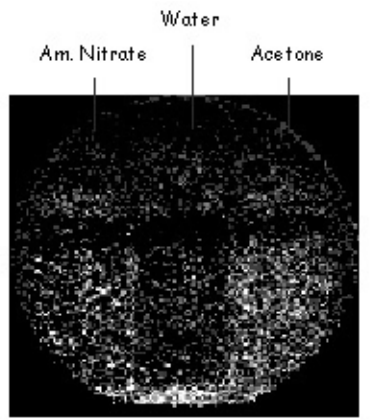

Carbon Mapp ing (areal density)

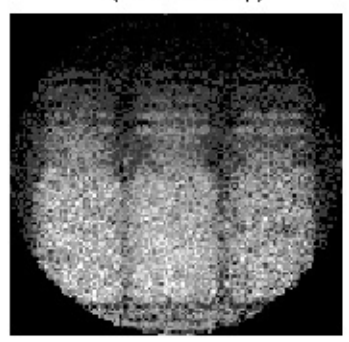

Oxygen Mapping (arealdensity)
Fig. 3. Elemental mapping images for three different materials. In each panel, the same three materials are shown (left to right): ammonium nitrate, water, and acetone. The four panels show the amount of (clockwise from top left) hydrogen, carbon, oxygen, and nitrogen present in each object, where brightness indicates high relative amounts of the individual element of interest. Intensity is not to scale between images.

Results of the imagery analysis show qualitative agreement between areal densities determined from the experiments and the expected values calculated based on the known elemental composition of the objects. As an example, Fig. 3 shows the radiographs of three materials - ammonium nitrate, water, and acetone - and their corresponding elemental densities calculated from Eq. 1 and attenuation coefficients experimentally derived from the calibration objects. These images clearly show a difference in the amount of each element present in the object, most noticeably in the hydrogen and nitrogen content, but the stark contrast in elemental density expected was not apparent, nor do the calculated elemental densities match the known elemental content of the objects. Furthermore, the quantitative agreement between experimental, simulated, and theoretically calculated values for the attenuation coefficients and areal densities was poor. The experimental values obtained for the attenuation coefficients significantly different both in value as well lacking in obvious effects from neutron cross section resonance features (for an example, see Fig. 4).

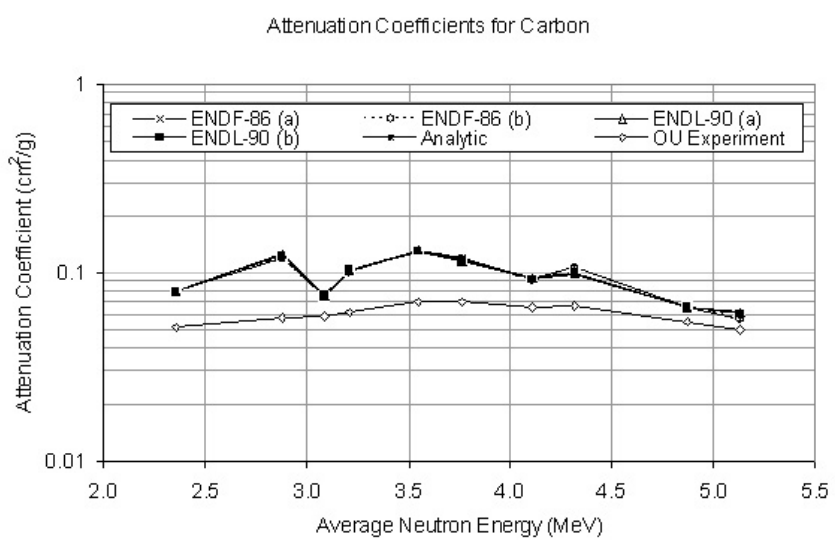

Fig. 4. Attenuation coefficients for carbon calculated from experimental results compared to accepted values. Note the similarity between all simulated and analytically-derived values and the discrepancy between those values and the experimentally-derived values. In addition, the lack of representation of the features of the cross-section for various neutron energies in the experimentally-derived attenuation coefficients is obvious.

The discrepancy between the expected and experimentally determined values for the areal density and attenuation coefficients is likely due to effects from gamma rays. Gamma rays are produced from deuterium impingement on materials in the gas target and accelerator beamline, as well as through neutron interactions in shielding materials. Gamma interactions in the scintillation material, designed to detect neutrons, also produce light which is detected by the CCD camera and is indistinguishable from the light produced by neutrons. Gamma rays of the same energy, in fact, produce factor of three to five more light than neutrons, obscuring the necessary differences in neutron detection at various energies required for elemental contrast in the final images [6]. Reduction of gamma contamination or a means to discriminate between gamma and neutron interactions is essential for accurate calculations of attenuation coefficients and subsequent areal densities based on pixel intensity.

In addition to gamma contamination, the difficulty of producing sufficient quantities of neutrons to allow for short, less than five minute, imaging times must be addressed. Longer imaging integration times result in more dark current and in the final images, degrading the quality of the images. Short irradiation times are also required for large-scale applications of NRR. Increasing the neutron flux can be accomplished in three ways: by increasing the deuteron current, by increasing the pressure in the deuterium gas cell, or a combination of the two. An increase in either the current or pressure of deuterium increases the mechanical and thermal stress on the gas target, which must be taken into account. Furthermore, increasing the gas pressure results in greater deuteron energy loss in the gas which broadens the energy spectrum of the neutrons produced.

\section{Solutions to Gamma Ray CONTAMination}

The ability of NRR to determine the elemental components of an object successfully rests on the ability to reduce the 
contaminating effects of gamma rays in the final images. Two methods have been devised to reduce the effects of gamma rays: a redesigned gas target intended to produce fewer gamma rays while simultaneously increasing the neutron flux, and the design of a neutron counting system, rather than direct imaging, for neutron detection and image formation. For direct imaging, the improved gas target will produce fewer overall gamma rays and a greatly increased (at least a factor of ten) neutron flux compared to the previous gas target used for NRR. While the gamma flux will not be eliminated entirely, lead shielding at the detector can remove many low-energy gamma rays and the increased neutron flux will result in decreased imaging times and greater contrast in the images, which will reduce the effect of contaminating gamma rays. The neutron counting method, used in conjunction with the redesigned gas target, exploits the difference in pulse height of gamma and neutron interactions in plastic scintillator to count only those interactions caused by neutrons.

\section{A. Gas Target}

The gas target consists of three main components: the gas cell, which holds the deuterium gas, the beam stop, which stops any incident deuterons that do not interact with the deuterium gas in the cell, and a set of beam scrapers, located upstream from the gas cell and designed to shape and center the deuteron beam into a beam spot $1 \mathrm{~cm}$ in diameter [7]. The gas cell is held at a pressure differential from the accelerator beam line vacuum by a thin window that must support both the pressure difference and heating from the deuteron beam. The beam stop ideally absorbs any deuterons that have not reacted with the deuterium in the gas cell without creating significant numbers of gamma rays or affecting the emitted neutrons.

Gamma production from deuterium impingement on metal in the accelerator beamline and gas target is the primary source of gamma production. To minimize the production of gamma rays in the target, a series of experiments was performed to determine optimal metals for gas target construction. We placed thin metal foils in front of a $2.5 \mathrm{MeV}$ deuteron beam to characterize the gamma spectra resulting from each metal and found tungsten, molybdenum and gold to produce the fewest overall and the least number of high energy gamma rays. In part based on these results and also due to its excellent thermal and structural properties, we designed the gas cell and the components of the gas target to ensure that all beam-facing components were made of tungsten.

The gas target was redesigned to take into account the need for increased neutron flux while simultaneously increasing the durability of the target. The gas target was designed to support a beam of $3 \mathrm{MeV}$ deuterons, (future testing may be done at this energy,) although most of the experiments and the testing of the gas target described here utilize a beam of $2.5 \mathrm{MeV}$ deuterons due to constraints on the accelerator system. A $1 \mu \mathrm{A}$ beam of $3 \mathrm{MeV}$ deuterons $1 \mathrm{~cm}$ in diameter will deposit 0.320 W of energy in the thin window. A support structure or "strongback" is provided for the thin entrance window in order to reduce the stresses that lead eventually to catastrophic failure or unacceptably high leak rates. This strongback consists of a $0.25 \mathrm{~cm}$ thick piece of tungsten with a circular pattern of holes $0.1 \mathrm{~cm}$ in diameter. This pattern allows for $60 \%$ transmission of the deuteron beam into the gas cell, but provides significant structural support and an extra heatremoval pathway. The same $3 \mathrm{MeV}, 1 \mu \mathrm{A}$ beam will deposit $1.269 \mathrm{~W}$ into the front strongback. These values are linearly dependent on beam current.

The metal beam stop of the gas target used in the experiments discussed above has been replaced in the new target design with a circulating argon gas beam stop. The argon chamber is separated from the gas cell by another $5 \mu \mathrm{m}$ thin tungsten window with a similar support structure. The argon is held at the same pressure as that of the deuterium in the cell, 4 atm, and thus does not apply any extra stress to the gas cell. The pumped argon provides an additional cooling mechanism for the gas cell as well as a reduction in the number of gamma rays produced by the slowing down and absorption of deuterons.

The gas target is designed to withstand a $3 \mathrm{MeV}$ deuteron beam at an average current of $50 \mu \mathrm{A}$ with the thin window and strongback arrangement and a gas cell pressure of $4 \mathrm{~atm}$. This configuration is expected to provide a neutron flux in excess of $10^{5} \mathrm{n} / \mathrm{cm}^{2} / \mathrm{s}$. This high neutron flux will increase the signalto-noise ratio, allow for longer imaging times, and improve the contrast ratios in the final images.

\section{B. Neutron/Gamma Discrimination}

Another critical component of the NRR system is the neutron detector and associated equipment used to generate images for analysis. Although commercially available solid plastic scintillator materials are expected to be adequate for NRR imaging with a cooled CCD camera with the implementation of the new high-flux gas target and gamma ray shielding, an alternative means of neutron detection neutron counting - is under consideration if gamma contamination of the image remains a problem. Neutron counting, as opposed to light integration as with imaging, allows discrimination between gamma and neutron signals based on pulse shape.

Fast neutrons are detected by hydrogen recoil; in other words, neutrons knock on hydrogen nuclei (protons) in the detector material and the recoil protons produce light through ionization [8]. The hydrogen rich scintillator is coupled with a photo multiplier tube (PMT) of matching spectral response through a light pipe. The electronic signal at the PMT anode is collected and electronically processed. Individual neutron and gamma ray events are recorded and digitized for discrete analysis of pulse height. Scintillation material has been obtained from Eljen Technology, Sweetwater, TX. For large objects, a row of one inch square scintillation arrays will be aligned and will be swept across large objects to produce resolved images.

Pulse shapes from liquid scintillators vary based on the energy and type of incoming particle. Some discrimination between neutrons and gamma-rays in the plastic scintillator can be obtained based on pulse height. Gamma discrimination for NRR, however, is complicated by the fact that high energy neutrons used for NRR have similar pulse heights to those of medium-energy gamma rays. The system that we have designed takes advantage of the discrepancy between the 
higher pulse heights of high-energy gamma rays compared to neutrons by setting a discrimination threshold to reject any pulses above a set limit, thereby discounting interactions due to high-energy gamma rays. Reduction of low energy gamma rays is accomplished using lead shielding to reduce the number of low energy gamma ray detections. This leaves only the realm where light pulses caused by high-energy neutrons blend with those caused by medium-energy gamma rays. Removing or reducing the effect of the high and low energy gammas should result in a significant reduction in the overall count rate and contrast-reducing gamma contamination of the images.

\section{CONCLUSIONS}

Neutron resonance radiography holds promise as a means to detect explosives in both checked luggage and air cargo. By using fast neutrons and their characteristic interactions with light elements such as carbon, nitrogen, oxygen, and hydrogen, the elemental content of concealed objects can be determined. NRR has the added advantage of using imaging techniques to determine the shapes of concealed objects; this, combined with elemental content, can lead to improved explosives detection without the need for intrusive searches. However, while simulations and simple experiments have demonstrated the viability of NRR, more complex experiments involving multiple elements and materials showed the need to address the problem of gamma-ray contamination of the beam. The cause of the discrepancy between expected values and experimental results in these latter experiments is likely to be gamma ray events in the plastic scintillator used for neutron detection. Two means of alleviating the gamma ray difficulty have been examined. Firstly, a new gas target has been designed as a neutron source to provide a high neutron flux while reducing gamma ray production in the gas target material. Secondly, neutron/gamma discrimination and neutron counting are under investigation as a way to reduce the number of gamma rays detected as significant events in the scintillator. These techniques can be implemented either separately or in conjunction with each other to improve the quality of the images and elemental resolution produced by NRR.

\section{ACKNOWLEDGMENT}

The authors would like to thank the staff of the Ohio University Accelerator Laboratory for their assistance. They would also like thank Ashley Finan and Jack Styczynski, as well as Gongyin Chen and Dave Perticone for many useful discussions.

The views and opinions presented in this document are those of the author(s) and do not necessarily represent the official position or policies of the US Government.

\section{REFERENCES}

[1] Krauss, R.A., "Signatures of Explosives by Elemental Composition Analysis". Proceedings of the Second Explosives Detection Technology Symposium \& Aviation Security Technology Conference, Atlantic City, NJ, pp. 18-25, W. Makky, Ed., Nov. 1996.

[2] G. Chen and R. Lanza, "Neutron Resonance Radiography for Elemental Imaging: Theory and Applications," IEEE Trans. Nucl. Sci., vol. 49, no. 4, pp. 1919-1924, Aug. 2002.

[3] J. I. W. Watterson and R. M. Ambrosi, "Some Fundamental Considerations in Resonance Imaging Using Fast Neutrons", Nuc. Inst. and Meth in Phys. A, vol. 513, pp. 367-373, 2002.

[4] J. Hall, "Monte Carlo modeling of neutron and gamma-ray imaging systems," in Proc. 5th Int. Conf. Applications of Nuclear Techniques, Crete, Greece, Bellingham, WA: SPIE, pp. 465-468, 1997, G. Vourvopoulos, Ed., June 1996.

[5] J. Guzek, "Elemental radiography using fast neutron beams,", Doctor of Philosophy dissertation, Univ. Witwatersrand, Witwatersrand, South Africa, Dec. 1999.

[6] Eljen Technology, Sweetwater, TX: http://www.eljentechnology.com.

[7] See W. Raas, et al., "Design and Testing of a High Pressure Gas Target for Fast Neutron Resonance Radiography", these proceedings.

[8] G. F. Knoll, Radiation Detection and Measurement, 3rd ed. John Wiley and Sons, Inc., New York, 2000, pp. 219-264. 